\title{
Efficient Excitation of Gain-Saturated Sub-9-nm-Wavelength Tabletop Soft-X-Ray Lasers and Lasing Down to $7.36 \mathrm{~nm}$
}

\author{
D. Alessi, ${ }^{1,2, *}$ Y. Wang, ${ }^{1,2}$ B. M. Luther, ${ }^{1,2}$ L. Yin, ${ }^{1,2}$ D. H. Martz, ${ }^{1,2}$ \\ M. R. Woolston, ${ }^{1,2}$ Y. Liu, ${ }^{1,3}$ M. Berrill,,${ }^{1,2,4}$ and J. J. Rocca ${ }^{1,2,5, \dagger}$ \\ ${ }^{1}$ NSF Engineering Research Center for Extreme Ultraviolet Science and Technology, Fort Collins, Colorado 80523, USA \\ ${ }^{2}$ Department of Electrical and Computer Engineering, Colorado State University, Fort Collins, Colorado 80523, USA \\ ${ }^{3}$ Department of Electrical Engineering and Computer Sciences, University of California Berkeley, Berkeley, California 94720, USA \\ ${ }^{4}$ Oak Ridge National Laboratory, Oak Ridge, Tennessee 37830, USA \\ ${ }^{5}$ Department of Physics, Colorado State University, Fort Collins, Colorado 80523, USA \\ (Received 16 September 2011; published 27 December 2011)
}

\begin{abstract}
We have demonstrated the efficient generation of sub-9-nm-wavelength picosecond laser pulses of microjoule energy at $1-\mathrm{Hz}$ repetition rate with a tabletop laser. Gain-saturated lasing was obtained at $\lambda=8.85 \mathrm{~nm}$ in nickel-like lanthanum ions excited by collisional electron-impact excitation in a precreated plasma column heated by a picosecond optical laser pulse of 4-J energy. Furthermore, isoelectronic scaling along the lanthanide series resulted in lasing at wavelengths as short as $\lambda=$ $7.36 \mathrm{~nm}$. Simulations show that the collisionally broadened atomic transitions in these dense plasmas can support the amplification of subpicosecond soft-x-ray laser pulses.
\end{abstract}

DOI: 10.1103/PhysRevX.1.021023

The high demand for bright soft-x-ray laser (SXRL) pulses greatly exceeds the beam time available at a few single-user free-electron laser facilities [1,2]. This motivates the development of more compact and widely accessible SXRLs for a broad range of experiments in small laboratory settings. Significant progress has been achieved in the past few years in the development of compact plasma-based soft-X-ray lasers [3-10]. However, repetitive operation of tabletop SXRLs has been limited to wavelengths above $10.9 \mathrm{~nm} \mathrm{[10].} \mathrm{At} \mathrm{shorter} \mathrm{wavelengths,} \mathrm{the}$ large pump energy required has limited the repetition rate to typically one shot per hour [11-15]. Soft-x-ray lasing at sub-10-nm wavelengths in lanthanide ions was first demonstrated using optical lasers of pump energy of several hundred joules $[12,13]$. Lasing in nickel-like lanthanum at $\lambda=8.85 \mathrm{~nm}$ was later obtained by using $18-\mathrm{J}$ pulses from a chirped-pulse-amplification (CPA) laser to achieve transient excitation, but with a gain-length product $(g \times l=7.7)$ that remained insufficient to reach gain saturation [15]. Progress toward saturated lasing in this transition has been recently reported [16,17]. In turn, lasing at $\lambda=7.36 \mathrm{~nm}$ in nickel-like Sm was initially demonstrated using 130-J pump pulses [12]. Later, gain saturation was reached using a picosecond-duration pump pulse with approximately $40 \mathrm{~J}$ of energy added to a prepulse of similar energy [14]. Also recently, the lasing threshold in this line

\footnotetext{
*dalessi@engr.colostate.edu
}

†jorge.rocca@colostate.edu

Published by the American Physical Society under the terms of the Creative Commons Attribution 3.0 License. Further distribution of this work must maintain attribution to the author(s) and the published article's title, journal citation, and DOI.
Subject Areas: Photonics, Plasma Physics

was reached through the use of a total optical pump energy of $36 \mathrm{~J}$ [18]. However, the practical realization of highrepetition-rate tabletop lasers at sub-10-nm wavelengths requires obtaining gain-saturated lasing at significantly lower pump energies. We report the generation of gainsaturated picosecond SXRL pulses at $\lambda=8.85 \mathrm{~nm}$ at $1-\mathrm{Hz}$ repetition rate. The result is obtained by using a picosecond pump pulse with an unprecedentedly low energy of $4 \mathrm{~J}$ and a total optical pump energy of $7.5 \mathrm{~J}$, that will make the operation at high-repetition rates possible. Furthermore, using the same pump energy, we observe lasing at wavelengths down to $\lambda=7.36 \mathrm{~nm}$ in transitions of higher- $Z$ nickel-like lanthanide ions, opening the prospect of practical gain-saturated tabletop lasers at shorter wavelengths. Modeling suggests that these dense-plasma amplifiers have the bandwidth necessary to sustain the amplification of sub-ps SXRL pulses.

We conduct hydrodynamic-atomic modeling and threedimensional ray-trace simulations for an axially uniform plasma column of a constant width, $30 \mu \mathrm{m}$. This model is coupled with a complete atomic code that is capable of solving for the atomic populations of all ion species in the relevant energy levels by using a fully transient solution, including full radiation transport. The results suggest that gain-saturated amplification in the $\lambda=8.85 \mathrm{~nm}, 4 d^{1} S_{0} \rightarrow$ $4{ }^{1} P_{1}$ line of nickel-like lanthanum can be achieved by using a total pump energy of only $4 \mathrm{~J}$. While the experimental realization can be expected to require larger pump energy to compensate for factors not considered in the model, for example, processes that decrease absorption at high irradiation intensities [19], imperfections in the plasma column uniformity and target oxide layer, its magnitude is likely to remain in the range that allows for a high-repetition-rate SXRL operation. A gain with a 
peak value of approximately $90 \mathrm{~cm}^{-1}$ and a duration of approximately 5 ps is computed to result from the irradiation of a solid lanthanum target with a sequence of a $3.3 \times$ $10^{12} \mathrm{~W} \mathrm{~cm}^{-2}$ prepulse, 210 ps FWHM in duration followed by traveling-wave excitation with a $2 \times$ $10^{14} \mathrm{~W} \mathrm{~cm}^{-2}, 3$-ps FWHM duration pulse impinging at a grazing incidence angle of $35^{\circ}$. The short pulse is shown by the computation to rapidly heat the region of the precreated plasma where the electron density is approximately $6 \times$ $10^{20} \mathrm{~cm}^{-3}$ to an electron temperature of approximately $850 \mathrm{eV}$. Ray-trace simulations predict that the SXRL pulse duration progressively shortens to reach $1.5-2$ ps just before gain saturation. As the pulse intensity continues to increase by an additional order of magnitude above saturation, the laser-pulse duration is computed to rebroaden to approximately $2.5 \mathrm{ps}$.

The SXRL results were obtained by irradiating 1-2-mmthick solid slab targets with a sequence of two laser pulses from a $\lambda=800 \mathrm{~nm}$ Ti:sapphire CPA laser consisting of a normal-incidence prepulse followed by a main picosecond pulse impinging at a grazing incidence angle of $35^{\circ}$ with a traveling-wave excitation velocity of $(1.04 \pm 0.03) c$. The pump laser has four stages of amplification, of which the last two are pumped with the frequency-doubled output of Nd-glass slab amplifiers designed to operate at repetition rates of up to $4 \mathrm{~Hz}$ [20]. The plasmas were created by normal-incidence irradiation at $I=6 \times 10^{12} \mathrm{~W} \mathrm{~cm}^{-2}$ with a 210-ps duration prepulse that is focused onto the target to form a line focus of an approximately $30 \mu \mathrm{m} \times$ $6.4 \mathrm{~mm}$ FWHM by using the combination of a spherical and a cylindrical lens. The plasma created by the prepulse is allowed to expand to reduce the density gradient and is subsequently rapidly heated by irradiation at $I=$ $6 \times 10^{14} \mathrm{~W} \mathrm{~cm}^{-2}$ with a 4-J pulse of a 1-3-ps duration shaped into a line focus of the same dimension. The target surface is tilted with respect to the axis of the short pulse to define a grazing incidence angle of $\theta=35^{\circ}$ for efficient heating [3,4]. At this angle of incidence, refraction couples the pump-beam energy into the plasma region where $n_{e}=5.7 \times 10^{20} \mathrm{~cm}^{-3}$. To assist in achieving efficient pumping, we developed a new focusing geometry designed to create a plasma column of constant width along the target [Fig. 1(a)]. In the conventional implementations of grazing incidence pumping, where a parabolic or spherical mirror is used to focus the beam $[3-5,9,10]$, the tilted target intercepts the short-pulse pump beam at different distances from the beam waist. This creates a "butterfly" shaped line focus on the target, which for small $f$-number systems, can deposit part of the pump energy outside the useful gain column, decreasing the gain. To overcome this limitation, we focus the short-pulse beam with a pair of cylindrical mirrors designed to create a line focus of constant width along a target irradiated at grazing incidence. The beam first impinges on a cylindrical mirror of $f=6.5 \mathrm{~m}$, which is used to generate a $6.4-\mathrm{mm}$-long line. The beam is

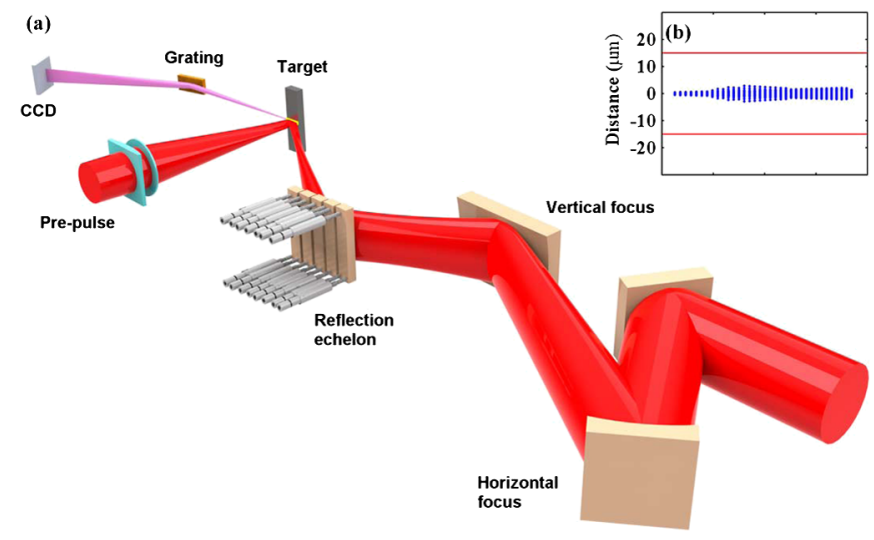

FIG. 1. Schematic representation of the setup used in the sub9-nm SXRL experiments. (a) A pair of cylindrical mirrors focuses the short-pulse beam at $35^{\circ}$ grazing incidence on the target, creating a line focus of uniform width. A reflection echelon is used to obtain quasi-traveling-wave excitation. (b) Ray-trace simulation of the line focus on target. All rays fall within the linewidth defined by diffraction (red lines).

subsequently made to impinge onto a second cylindrical mirror of $f=2.8 \mathrm{~m}$, which generates a $30-\mu \mathrm{m}$-wide vertical focus. Each segment of the converging beam impinging on the vertical-focus mirror has a unique focal length $f=R /(2 \cos \theta)$ and a unique path length to the tilted target. The difference between path length and focal length is brought below the diffraction limit by using an incidence angle of $54^{\circ}$ on the vertical mirror [see the ray-trace simulations results in Fig. 1(b)].

Because of the short duration of the gain, the mismatch between the propagation velocities of the pump pulse and the amplified pulse significantly reduces the amplification of the SXRL pulse. To overcome this limitation, a reflection echelon $[21,22]$ composed of five mirror segments was used to obtain quasi-traveling-wave excitation. Measurements at the $1-\mathrm{Hz}$ repetition rate are obtained continuously by moving the target at a speed of $200 \mu \mathrm{m} / \mathrm{s}$ to renew the surface after each shot. The output of the SXRLs was analyzed by using a flat-field spectrometer with a nominally 1200 -lines $/ \mathrm{mm}$ grating set at $3^{\circ}$ grazing incidence and a back-illuminated CCD detector placed at $48 \mathrm{~cm}$ from the target. Zirconium filters with thickness up to $2.1 \mu \mathrm{m}$ were used to avoid saturating the detector and to eliminate visible plasma light. The SXRL-pulse energy was estimated from the CCD counts taking into account the attenuation of the filters, the grating efficiency, and the quantum efficiency of the detector.

Figure 2(a) shows a series of on-axis spectra as a function of the length of a lanthanum plasma column created by depositing $4 \mathrm{~J}$ of short-pulse energy and a total of 7.5-J optical pump pulse energy on target at the irradiation conditions described above. Strong amplification is observed in the $\lambda=8.85 \mathrm{~nm}, 4 d^{1} S_{0} \rightarrow 4 p^{1} P_{1}$ transition of Ni-like La. Figure 2(b) shows that the intensity grows by nearly 4 orders of magnitude as the plasma length increases 
(a)
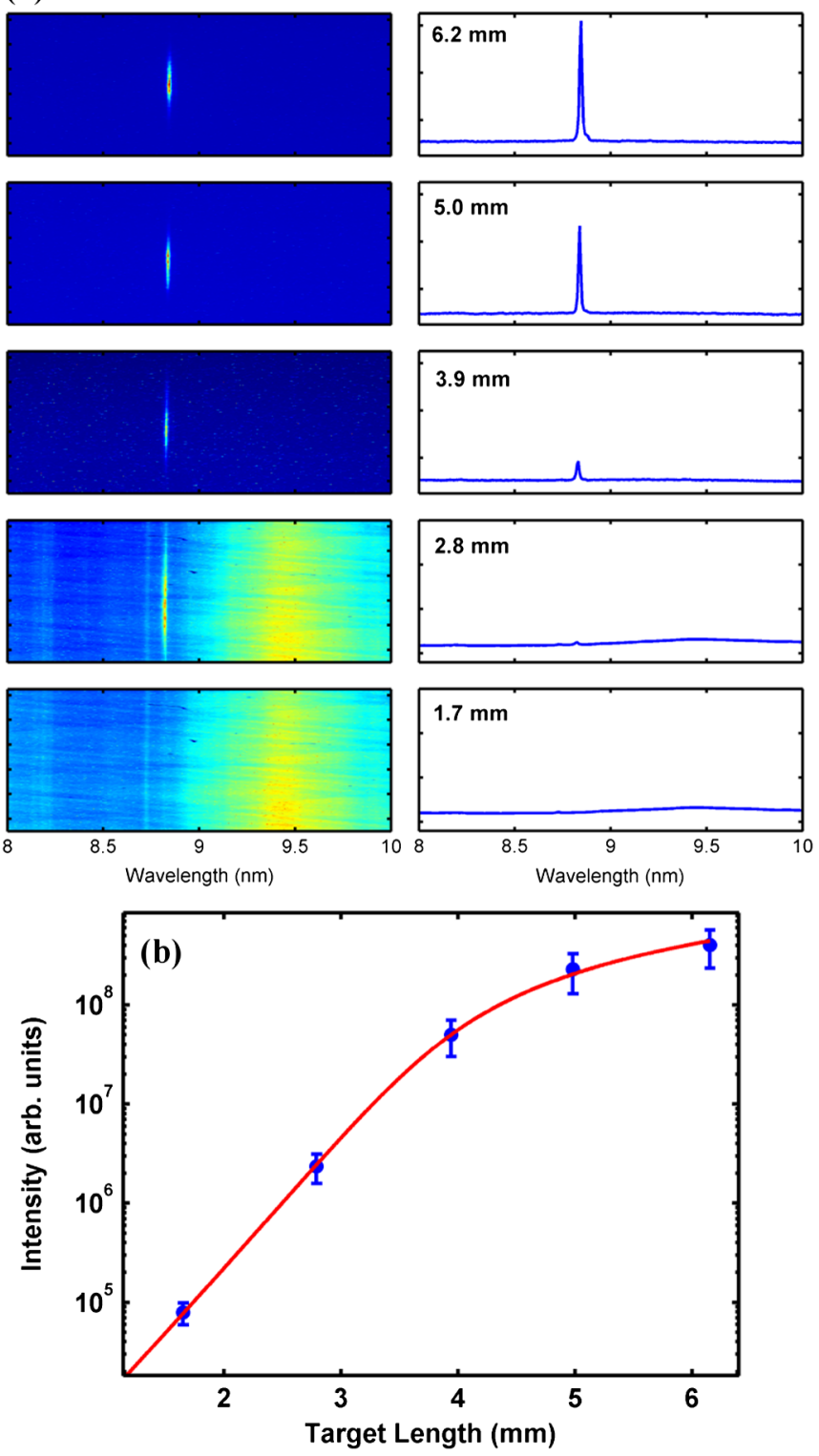

FIG. 2. (a) End-on spectra of a line-focus lanthanum plasma column showing saturated amplification in the $\lambda=8.85 \mathrm{~nm}$ line of Ni-like La. (b) Intensity of the $\lambda=8.85 \mathrm{~nm}$ laser line as a function of the plasma-column length. The line is a fit of the data that yields a gain coefficient of $33 \mathrm{~cm}^{-1}$ and a gain-length product of 14.6. The points are the average of 10 laser shots and the error bar represents 1 standard deviation.

from 1.7 to $6.2 \mathrm{~mm}$. Saturation of the intensity is observed to have an onset at a plasma-column length of approximately $4 \mathrm{~mm}$. A fit of the data with an expression that takes into account gain saturation [23] yields a gain coefficient of $33 \mathrm{~cm}^{-1}$ and a gain-length product of 14.6. The energy of the most intense laser pulses was estimated to be $2.7 \mu \mathrm{J}$ from the CCD counts.

The gain-saturated operation was confirmed by measuring the SXRL flux at the exit of the amplifier. The near-field profile of the $\lambda=8.85 \mathrm{~nm}$ laser is recorded by imaging the output of the plasma amplifier with a $f=$ $25 \mathrm{~cm}$ near-normal-incidence Mo-Y multilayer mirror onto a back-illuminated CCD detector. The mirror was measured at a synchrotron beam line to have a reflectivity of $25 \%$ at this wavelength. Figure 3(a) shows that the beam pattern at the amplifier exit consists of a spot with dimensions of approximately $19 \mu \mathrm{m} \times 30 \mu \mathrm{m}$ FWHM in the directions perpendicular and parallel to the target, respectively, centered at $14 \mu \mathrm{m}$ from the target surface. This area defines a laser fluence of approximately $0.6 \mathrm{~J} \mathrm{~cm}^{-2}$ for the most intense laser shots. By assuming the laser-pulse duration of $2.5 \mathrm{ps}$ from the simulations, the laser intensity at the amplifier exit is estimated to be $2.4 \times 10^{11} \mathrm{~W} \mathrm{~cm}^{-2}$,
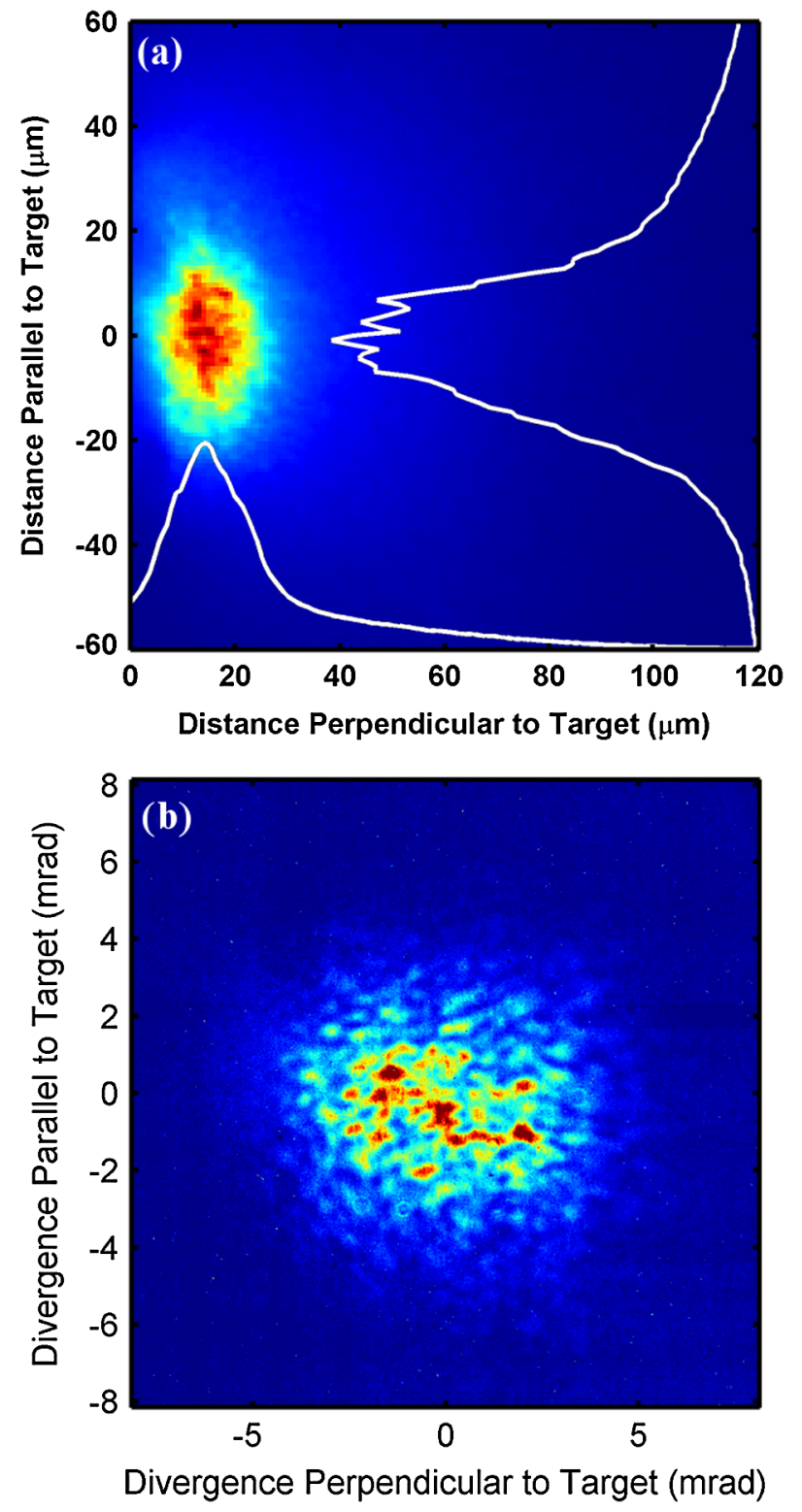

FIG. 3. Characteristics of the $\lambda=8.85 \mathrm{~nm}$ lanthanum laser. (a) Measured near-field pattern. The center of the laser beam at the amplifier exit is $14 \mu \mathrm{m}$ from the target. (b) Measured far-field pattern at a distance of $0.83 \mathrm{~m}$ from the source. 
which significantly exceeds the saturation intensity of $(1.5-3) \times 10^{10} \mathrm{~W} \mathrm{~cm}^{-2}$, computed by using a detailed atomic model with atomic rates from the Flexible Atomic Code [24]. This confirms that the laser operates in gain saturation. The profile of the far-field beam is recorded on a CCD by bending the beam with a $45^{\circ}$ incidence angle narrow-band Mo-Y multilayer mirror that also serves to filter background plasma light. Far-field images acquired at $0.86 \mathrm{~m}$ from the amplifier exit, such as that illustrated in Fig. 3(b), show the laser beam divergence is $(5.9 \pm 0.7) \times(4.7 \pm 0.7) \mathrm{mrad}^{2}$ in the directions perpendicular and parallel to the target surface, respectively.

Figure 4(b) illustrates the variations in the intensity of the $\lambda=8.8 \mathrm{~nm}$ laser pulses for 10 consecutive shots recorded at $1-\mathrm{Hz}$ repetition rate, with the target moving at a velocity of $200 \mu \mathrm{m} / \mathrm{s}$. Figure 4(a) shows that the maximum laser output is observed when the short pulse arrives at the target approximately 25 ps before the maximum of the 210-ps FWHM prepulse. This implies that more than half of the prepulse energy is not used in the laserexcitation process. Therefore, optimization of the prepulse should result in a further reduction of the total pump energy required for saturated operation. The combination of these results with future advances in diode-pumped CPA pump lasers $[25,26]$ that have already reached 1-J energy output at $10-\mathrm{Hz}$ repetition rate [25], promise to lead to the realization of high-average-power sub-9-nm lasers on a tabletop (e.g., $0.25 \mathrm{~mW}$ at $100 \mathrm{~Hz}$ ).

Another significant aspect of these amplifiers is the fact that collisional SXRL amplification at shorter wavelengths favors higher plasma densities, resulting in collisionally broadened lines that can support the amplification of subpicosecond seed XRL pulses, a subject of current interest

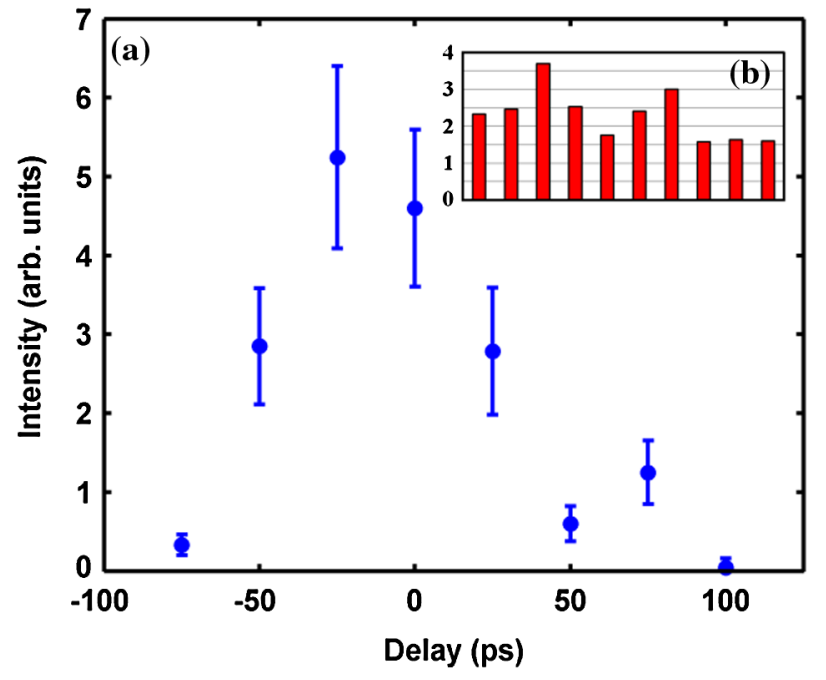

FIG. 4. (a) Measured laser intensity as a function of the delay between the peak of the prepulse and the short pulse. The points are the average of 4-15 laser shots and the error bar represents 1 standard deviation. (b) Measured shot-to-shot intensity variation of the $\lambda=8.85 \mathrm{~nm}$ laser operating at a $1-\mathrm{Hz}$ repetition rate.
[27-29]. Model simulations indicate that amplification at $\lambda=8.85 \mathrm{~nm}$ occurs in a plasma region where the plasma density is (5-9) $\times 10^{20} \mathrm{~cm}^{-3}$ and the ion temperature is approximately $150 \mathrm{eV}$. At the plasma conditions for peak gain, the profile of the laser line is defined by collisional broadening $\left(\Delta \nu_{L}=1.7 \times 10^{12} \mathrm{~Hz}\right)$ and Doppler contributions $\left(\Delta \nu_{D}=2.8 \times 10^{12} \mathrm{~Hz}\right)$ that define a Voigt linewidth of $\Delta \nu=3.8 \times 10^{12} \mathrm{~Hz}$. The variation of the bandwidth and the duration of an injected high-harmonic seed pulse as it propagates through the lanthanum plasma amplifier was computed with a 3D ray-trace post processor that couples the amplification and ray propagation equations with a time- and space-dependent atomic-physics model that includes all relevant levels and stimulated emission. The ray-trace code is capable of fully resolving the temporal duration and the bandwidth of both the amplified stimulated emission and the amplified seed beam. While this code does not treat the nonadiabatic variation of the electric dipole induced by the external radiation as MaxwellBloch codes do [30,31], it employs a fully coupled atomic model and 3D propagation that allows the simulation of amplified seed-pulse characteristics that are in good agreement with experiments $[28,32,33]$. Pulse broadening of the seed, due to line narrowing, is included by using the Fourier transform of the amplified bandwidth and accounting for the group-velocity delay caused by the gain profile. Figure 5 shows the computed pulsewidth variation of an injected $\lambda=8.85 \mathrm{~nm}, 30$-fs duration high-harmonic seed pulse as it propagates thought the lanthanum plasma amplifier for plasma conditions that result in an amplified spontaneous emission (ASE) laser output energy similar

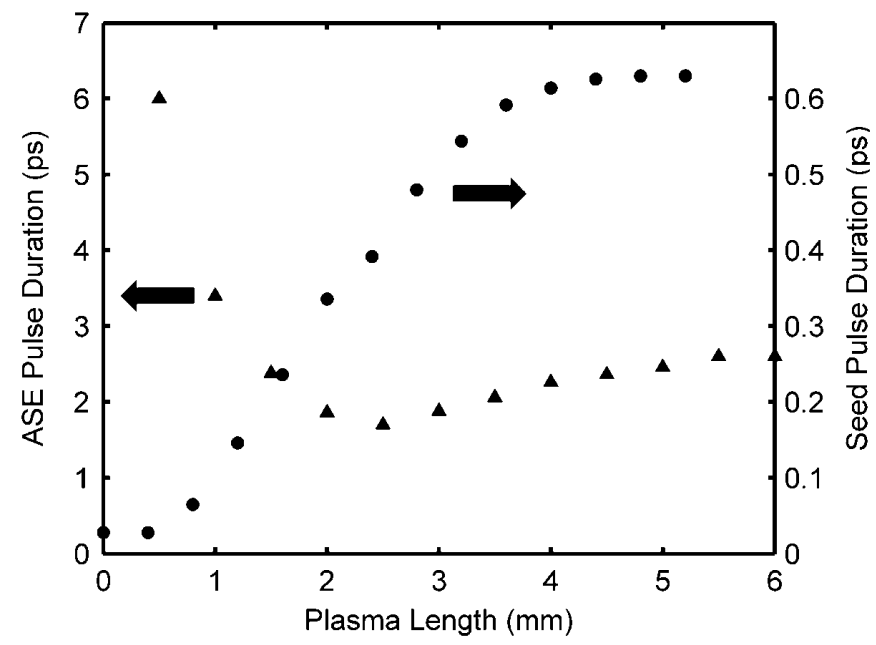

FIG. 5. Computed variation of the FWHM pulse duration of the $\lambda=8.85 \mathrm{~nm}$ ASE laser (triangles), and amplified highharmonic seed pulse (circles) as a function of the lanthanum plasma column length. The seed-pulse duration initially increases due to linewidth narrowing and later, by saturation broadening. The high-harmonic seed pulse is assumed to have an energy of $0.1 \mathrm{~nJ}$ and the gain and plasma conditions are those resulting from the simulations described in the text. 

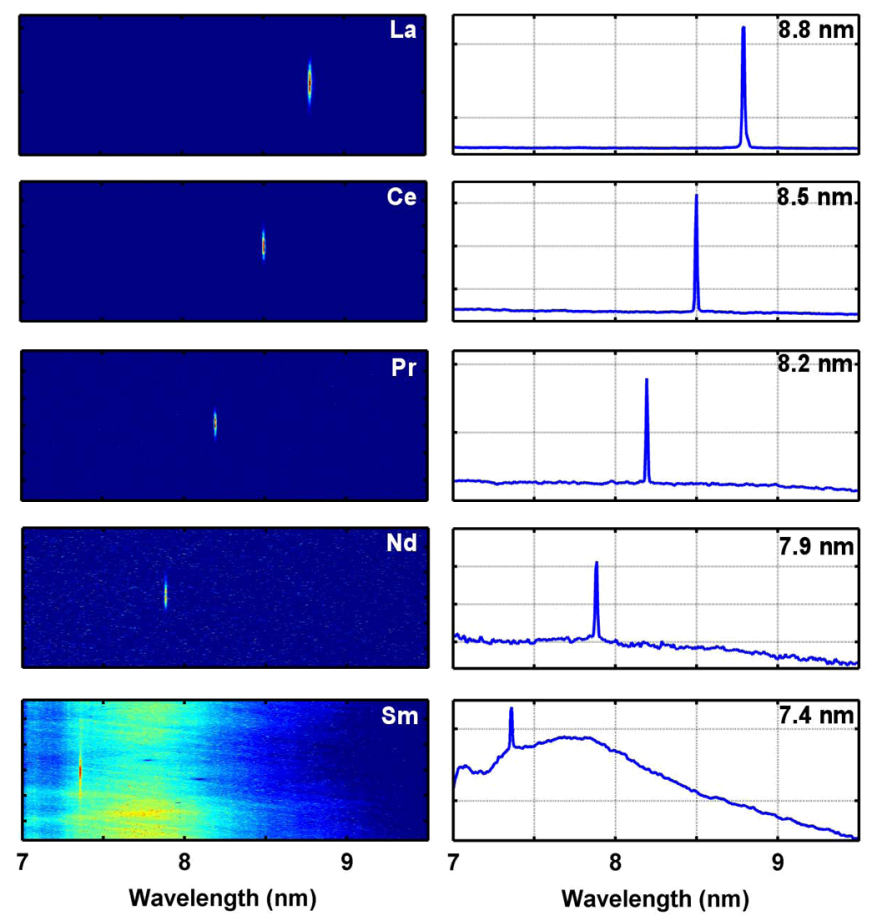

FIG. 6. End-on spectra showing lasing at progressively shorter wavelengths in the $4 d^{1} S_{0} \rightarrow 4 p^{1} P_{1}$ line of nickel-like lanthanide ions, down to $\lambda=7.36 \mathrm{~nm}$ in nickel-like samarium.

to that obtained in the experiment. Simulations conducted for a range of different pumping parameters predict outputpulse durations in the range of 500-700 fs and amplified seed-pulse energies of up to $0.1 \mu \mathrm{J}$. The computed variation of the pulsewidth of the unseeded ASE laser as a function of plasma-column length is also shown in Fig. 5.

The demonstration of a gain-saturated tabletop laser at $\lambda=8.85 \mathrm{~nm}$ with low pump energy also opens the prospect for bright high-repetition-rate plasma-based lasers at shorter wavelengths. In progress toward this goal we made use of isoelectronic scaling along the elements of the lanthanide series to obtain lasing in several shorter wavelength transitions. The spectra of Fig. 6 shows that the use of similar irradiation conditions resulted in lasing at $\lambda=8.5 \mathrm{~nm}$ in Ni-like Ce, $8.2 \mathrm{~nm}$ in Ni-like Pr, and $7.9 \mathrm{~nm}$ in Ni-like Nd. Lasing was also observed at $7.36 \mathrm{~nm}$ in Ni-like $\mathrm{Sm}$ by reducing the duration of the main pump pulse to 1.1 ps. The laser emission at $8.5 \mathrm{~nm}$ in Ni-like Ce is very strong. From comparison with the $\lambda=8.85 \mathrm{~nm}$ amplifier measurements, the $8.5-\mathrm{nm}$ laser is estimated to be operating at, or close to, gain saturation.

In summary, we have demonstrated for the first time the generation of bright gain-saturated soft-x-ray laser pulses of sub-9-nm wavelengths with a tabletop laser operating at $1-\mathrm{Hz}$ repetition rate. Using a picosecond optical laser pump with an energy of $4 \mathrm{~J}$, we have also obtained laser amplification at wavelengths as short as $\lambda=7.36 \mathrm{~nm}$. The combination of these results with new advances in high-repetition-rate diode-pumped optical lasers promises to lead to the generation of laser beams of high average power and sub-10-nm wavelengths on a tabletop. The short wavelength, microjoule pulse energy, picosecond pulse duration, and repetitive operation of these lasers will enable new applications such as sequential imaging of ultrafast nanoscale dynamic phenomena to be realized on a tabletop.

The authors thank Eric Gullikson and David Attwood for their contributions to the development of the $\lambda=8.85 \mathrm{~nm}$ soft-x-ray multilayer mirrors used to diagnose the laser beam; E. Cabosh, D. Reinholz, C. Menoni and D. Patel for their contributions to the development of the pumpoptics design and fabrication; and L. Durivage for his assistance with model computations. This work was supported by the NSF ERC for Extreme Ultraviolet Science and Technology under NSF Grant No. EEC 0310717 and by the AMOS program of the Chemical Sciences, Geosciences, and Biosciences Division, Office of Basic Energy Sciences, U.S. Department of Energy using equipment developed under NSF Grant No. MRI-ARRA 09-561. M. B. acknowledges support for staff members at Oak Ridge National Laboratory managed by UT-Battelle, LLC, for the U.S. Department of Energy under Contract No. DE-AC05-00OR22725. Model computations were partially done using the CSU ISTeC Cray HPC System supported by NSF Grant No. CNS-0923386.

[1] W. Ackermann, G. Asova, V. Ayvazyan, A. Azima, N. Baboi, J. Bähr, V. Balandin, B. Beutner, A. Brandt, A. Bolzmann, et al., Operation of a Free-Electron Laser from the Extreme Ultraviolet to the Water Window, Nat. Photon. 1, 336 (2007).

[2] B. W. J. McNeil and N.R. Thompson, X-Ray FreeElectron Lasers, Nat. Photon. 4, 814 (2010).

[3] R. Keenan, J. Dunn, P. K. Patel, D. F. Price, R. F. Smith, and V.N. Shlyaptsev, High-Repetition-Rate GrazingIncidence Pumped X-Ray Laser Operating at $18.9 \mathrm{~nm}$, Phys. Rev. Lett. 94, 103901 (2005).

[4] B. M. Luther, Y. Wang, M. A. Larotonda, D. Alessi, M. Berrill, M. C. Marconi, J. J. Rocca, and V. N. Shlyaptsev, Saturated High-Repetition-Rate 18.9-nm Tabletop Laser in Nickellike Molybdenum, Opt. Lett. 30, 165 (2005).

[5] Y. Wang, M. A. Larotonda, B. M. Luther, D. Alessi, M. Berrill, V. N. Shlyaptsev, and J. J. Rocca, Demonstration of High-Repetition-Rate Tabletop Soft-X-Ray Lasers with Saturated Output at Wavelengths Down to $13.9 \mathrm{~nm}$ and Gain Down to 10.9 nm, Phys. Rev. A 72, 053807 (2005).

[6] T. Mocek, C. M. McKenna, B. Cros, S. Sebban, D. J. Spence, G. Maynard, I. Bettaibi, V. Vorontsov, A.J. Gonsavles, and S. M. Hooker, Dramatic Enhancement of XUV Laser Output Using a Multimode Gas-Filled Capillary Waveguide, Phys. Rev. A 71, 013804 (2005).

[7] H. T. Kim, I. W. Choi, N. Hafz, J. H. Sung, T. J. Yu, K. H. Hong, T. M. Jeong, Y. C. Noh, D. K. Ko, K. A. Janulewicz, J. Tummler, P. V. Nickles, W. Sandner, and J. Lee, 
Demonstration of a Saturated Ni-Like Ag X-Ray Laser Pumped by a Single Profiled Laser Pulse from a 10-Hz Ti: sapphire Laser System, Phys. Rev. A 77, 023807 (2008).

[8] M. Nishikino, N. Hasegawa, T. Kawachi, H. Yamatani, K. Sukegawa, and K. Nagashima, Characterization of a High Brilliance Soft X-Ray Laser at $13.9 \mathrm{~nm}$ by Use of an Oscillator-Amplifier Configuration, Appl. Opt. 47, 1129 (2008).

[9] M. Grunig, C. Imesch, F. Staub, and J.E. Balmer, Saturated X-Ray Lasing in Ni-Like Sn at $11.9 \mathrm{~nm}$ Using the GRIP Scheme, Opt. Commun. 282, 267 (2009).

[10] D. Alessi, D. H. Martz, Y. Wang, M. Berrill, B. M. Luther, and J. J. Rocca, Gain-Saturated $10.9 \mathrm{~nm}$ Tabletop Laser Operating at $1 \mathrm{~Hz}$ Repetition Rate, Opt. Lett. 35, 414 (2010).

[11] B. J. Mac Gowan, L. B. da Silva, D. J. Fields, C. J. Keane, J. A. Koch, R. A. London, D. J. Matthews, S. Maxon, A. L. Osterheld, J. H. Scofiled, G. Shinkaveg, J. E. Trebes, and R.S Walling, Short Wavelength Laser Research at Lawrence Livermore National Laboratory, Phys. Fluids B 4, 2326 (1992).

[12] H. Daido, Y. Kato, K. Murai, S. Ninomiya, R. Kodame, G. Yuan, Y. Oshikane, M. Takagi, H. Takabe, and F. Koike, Efficient Soft X-Ray Lasing at 6-8 $\mathrm{nm}$ in Nickel-like Lanthanides, Phys. Rev. Lett. 75, 1074 (1995).

[13] H. Daido, S. Ninomiya, T. Imani, R. Kodama, M. Takagi, Y. Kato, K. Murai, J. Zhang, Y. You, and Y. Gu, Nickellike Soft-X-Ray Lasing at the Wavelengths Between 14 and 7.9 nm, Opt. Lett. 21, 958 (1996).

[14] J. Zhang, A. G. MacPhee, J. Lin, E. Wolfrum, R. Smith, C. Danson, M. H. Key, C. L.S. Lewis, D. Neely, J. Nilsen, G. J. Pert, G. J. Tallents, and J. S. Wark, A Saturated X-Ray Laser Beam at 7 Nanometers, Science 276, 1097 (1997).

[15] T. Kawachi, A. Sasaki, M. Tanaka, M. Kishimoto, N. Hasegawa, K. Nagashima, M. Koike, H. Daido, and Y. Kato, Observation of Strong Soft-X-Ray Amplification at $8.8 \mathrm{~nm}$ in the Transient Collisional-Excitation Scheme, Phys. Rev. A 69, 033805 (2004).

[16] J. E. Balmer, F. Staub, C. Imesch, and D. Bleiner, Sub-10$n m$ Wavelength Ni-Like Ion X-Ray Lasers, Proc. SPIE Int. Soc. Opt. Eng., 8140, 81400X (2011).

[17] J. J. Rocca Y. Wang, D. Alessi, B. A. Reagan, B. M. Luther, A. H. Curtis, K. Wernsing, F. Furch, M. R. Woolston, D. H. Martz, V. N. Shlyaptsev, and M. Berrill, Advances in High Repetition Rate Table-Top Soft X-Ray Lasers, Proc. SPIE Int. Soc. Opt. Eng., 8140, 81400I (2011).

[18] D. Zimmer, D. Ros, O. Guilbaud, J. Habib, S. Kazamias, B. Zielbauer, V. Bagnoud, B. Ecker, D. C. Hochhaus, B. Aurand, P. Neumayer, and T. Kuehl, Short-Wavelength Soft-X-Ray Laser Pumped in Double-Pulse Single-Beam Non-normal Incidence, Phys. Rev. A 82, 013803 (2010).

[19] R. E. King, G. J. Pert, S. P. McCabe, P. A. Simms, A. G. MacPhee, C. L. S. Lewis, R. Keenan, R. M. N. O’Rourke, G. J. Tallents, S. J. Pestehe, F. Strati, D. Neely, and R. Allott, Saturated X-Ray Lasers at 196 and 73 A Pumped by a Picosecond Traveling-Wave Excitation, Phys. Rev. A 64, 053810 (2001).

[20] D. Martz, D. Alessi, B. M. Luther, Y. Wang, D. Kemp, M. Berrill, and J. J. Rocca, High Energy 13.9 nm Table-Top
Soft X-ray Laser at $2.5 \mathrm{~Hz}$ Repetition Rate Excited by a Slab-Pumped Ti:sapphire Laser, Opt. Lett. 35, 1632 (2010).

[21] J. Dunn, Y. Li, A. L. Osterheld, J. Nilsen, J. R. Hunter, and V. N. Shlyaptsev, Gain Saturation Regime for Tabletop, Ni-Like Ion Transient X-Ray Lasers, Phys. Rev. Lett. 84, 4834 (2000).

[22] J. R. Crespo Lopez-Urrutia and E. E. Fill, Traveling-Wave Excitation of an X-Ray Laser Medium, Proc. SPIE Int. Soc. Opt. Eng. 2012, 258 (1994).

[23] G. J. Tallents, Y. Abou-Ali, M. Edwards, R. E. King, G. J. Pert, S. J. Pestehe, F. Strati, R. Keenan, C. L. S. Lewis, S. Topping, O. Guilbaud, A. Klisnick, D. Ros, R. Clarke, D. Neely, and M. Notley, in X-Ray Lasers 2002: 8th International Conference on X-Ray Lasers, edited by J. J. Rocca, J. Dunn, and S. Suckewer, AIP Conference Proceedings, No. C641 (AIP, New York, 2002), p. 291.

[24] M.F. Gu, in Atomic Processes in Plasmas: 14th APS Topical Conference on Atomic Processes in Plasmas, edited by J.S. Cohen, S. F. Mazevet, and D. P. Kilcrease, AIP Conf. Proc. Vol. 730 (AIP, New York, 2004) pp. 127.

[25] F. Furch, B. Reagan, B. Luther, A. Curtis, S. Meehan, and J. J. Rocca, Demonstration of an All-Diode-Pumped Soft X-Ray Laser, Opt. Lett. 34, 3352 (2009).

[26] J. Tümmler, R. Jung, H. Stiel, P. V. Nickles, and W. Sandner, High-Repetition-Rate Chirped-PulseAmplification Thin-Disk Laser System with Joule-Level Pulse Energy, Opt. Lett. 34, 1378 (2009).

[27] P. Zeitoun, G. Faivre, S. Sebban, T. Mocek, A. Hallou, M. Fajardo, D. Aubert, Ph. Balcou, F. Burgy, D. Douillet, S. Kazamias, G. de Lachèze-Murel, T. Lefrou, S. le Pape, P. Mercère, H. Merdji, A. S. Morlens, J. P. Rousseau, and C. Valentin, A High-Intensity Highly Coherent Soft X-Ray Femtosecond Laser Seeded by a High Harmonic Beam, Nature (London) 431, 426 (2004).

[28] Y. Wang, E. Granados, F. Pedaci, D. Alessi, B. Luther, M. Berrill, and J. J. Rocca, Phase-Coherent, Injection-Seeded, Table-Top Soft-X-Ray Lasers at $18.9 \mathrm{~nm}$ and $13.9 \mathrm{~nm}$, Nat. Photon. 2, 94 (2008).

[29] C. M. Kim, K. A. Janulewicz, H. T. Kim, and J. Lee, Amplification of a High Harmonic Pulse in an Active Medium of a Plasma-Based Soft X-Ray Laser, Phys. Rev. A 80, 053811 (2009).

[30] I. R. Al'miev, O. Larroche, D. Benredjem, J. Dubaut, S. Kazamias, C. Moller, and A. Klisnick, Dynamical Description of Transient X-Ray Lasers Seeded with High Order Harmonic Radiation through Maxwell-Bloch Numerical Simulations, Phys. Rev. Lett. 99, 123902 (2007).

[31] C. M. Kim, J. Lee, and K. A. Jenulewicz, Coherent Amplification of an Ultrshort Pulse in a High- and Swept-Gain Medium with Level Degeneracy, Phys. Rev. Lett. 104, 053901 (2010).

[32] Y. Wang, M. Berrill, F. Pedaci, M. M. Shakya, S. Gilbertson, Z. Chang, E. Granados, B. M. Luther, M. A. Larotonda, and J. J. Rocca, Measurement of 1 Picosecond Soft X-Ray Laser Pulses from an Injection-Seeded Plasma Amplifier, Phys. Rev. A 79, 023810 (2009).

[33] Y. Wang, E. Granados, M. A. Larotonda, M. Berrill, B. M. Luther, D. Patel, C. S. Menoni, and J. J. Rocca, HighBrightness Injection-Seeded Soft-X-Ray-Laser Amplifier Using a Solid Target, Phys. Rev. Lett. 97, 123901 (2006). 